\title{
Multiple functional polymorphisms in the G6PC2 gene contribute to the association with higher fasting plasma glucose levels
}

\author{
D. A. Baerenwald • A. Bonnefond • N. Bouatia-Naji • \\ B. P. Flemming • O. C. Umunakwe • J. K. Oeser $\cdot$ L. D. Pound • \\ N. L. Conley • S. Cauchi - S. Lobbens • E. Eury • B. Balkau • \\ O. Lantieri • MAGIC Investigators • P. K. Dadi • \\ D. A. Jacobson • P. Froguel • R. M. O'Brien
}

Received: 19 October 2012 / Accepted: 28 January 2013 /Published online: 19 March 2013

(C) Springer-Verlag Berlin Heidelberg 2013

\begin{abstract}
Aims/hypothesis We previously identified the G6PC2 locus as a strong determinant of fasting plasma glucose (FPG) and showed that a common $G 6 P C 2$ intronic single nucleotide polymorphism (SNP) (rs560887) and two common G6PC2 promoter SNPs (rs573225 and rs13431652) are highly associated with FPG. However, these promoter SNPs have complex effects on $G 6 P C 2$ fusion gene expression, and our data suggested that only rs 13431652 is a potentially
\end{abstract}

D. A. Baerenwald, A. Bonnefond and N. Bouatia-Naji contributed equally to this study.

Electronic supplementary material The online version of this article (doi:10.1007/s00125-013-2875-3) contains peer-reviewed but unedited supplementary material, which is available to authorised users.

D. A. Baerenwald · B. P. Flemming · O. C. Umunakwe •

J. K. Oeser $\cdot$ L. D. Pound $\cdot$ N. L. Conley $\cdot$ P. K. Dadi •

D. A. Jacobson · R. M. O’Brien ( $\varangle)$

Department of Molecular Physiology and Biophysics,

Vanderbilt University School of Medicine,

8415 MRB IV, 2213 Garland Avenue,

Nashville, TN 37232-0615, USA

e-mail: richard.obrien@vanderbilt.edu

A. Bonnefond $\cdot$ N. Bouatia-Naji $\cdot$ S. Cauchi $\cdot$ S. Lobbens $\cdot$

E. Eury $\cdot$ P. Froguel

CNRS-UMR-8199, Institut Pasteur de Lille,

Lille, France

A. Bonnefond $\cdot$ N. Bouatia-Naji $\cdot$ S. Cauchi $\cdot$ S. Lobbens $\cdot$

E. Eury $\cdot$ P. Froguel

University Lille, Nord de France, Lille, France

N. Bouatia-Naji

Inserm U970, Paris Cardiovascular Research Center PARCC,

Paris, France causative SNP. Here we examine the effect of rs560887 on G6PC2 pre-mRNA splicing and the contribution of an additional common G6PC2 promoter SNP, rs 2232316 , to the association signal.

Methods Minigene analyses were used to characterise the effect of rs560887 on G6PC2 pre-mRNA splicing. Fusion gene and gel retardation analyses characterised the effect of rs 2232316 on $G 6 P C 2$ promoter activity and transcription factor binding. The genetic association of rs2232316 with

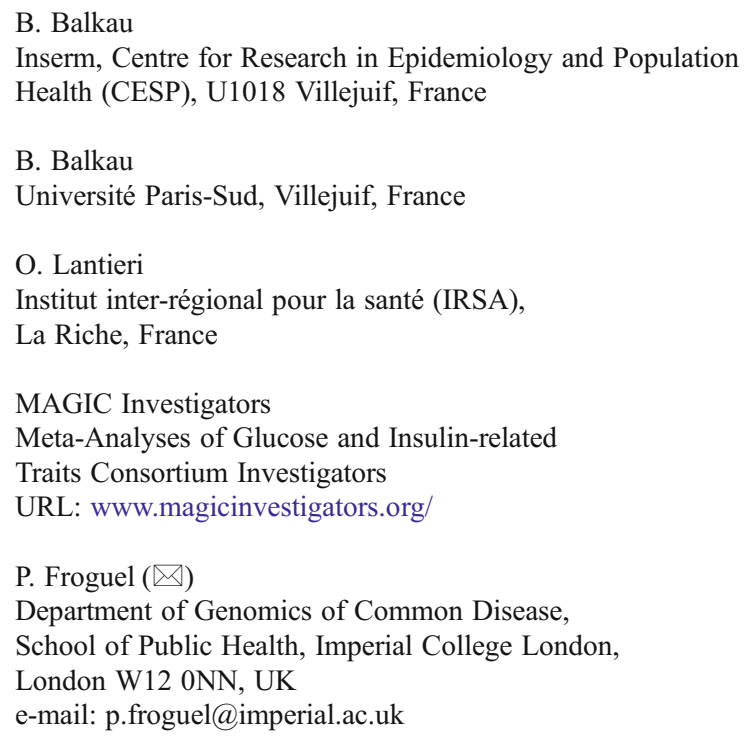


FPG variation was assessed using regression adjusted for age, sex and BMI in 4,220 Europeans with normal FPG.

Results The rs560887-G allele was shown to enhance G6PC2 pre-mRNA splicing, whereas the rs2232316-A allele enhanced $G 6 P C 2$ transcription by promoting Foxa2 binding. Genetic analyses provide evidence for association of the rs2232316-A allele with increased FPG $\left(\beta=0.04 \mathrm{mmol} / 1 ; p=4.3 \times 10^{-3}\right)$ as part of the same signal as rs560887, rs573225 and rs13431652.

Conclusions/interpretation As with rs13431652, the in situ functional data with rs560887 and rs2232316 are in accord with the putative function of $G 6 P C 2$ in pancreatic islets, and suggest that all three are potentially causative SNPs that contribute to the association between $G 6 P C 2$ and FPG.

Keywords Beta cell · Fasting plasma glucose - G6PC2/IGRP . Pancreatic islet $\cdot$ Splicing $\cdot$ Transcription

$\begin{array}{ll}\text { Abbreviations } \\ \text { DESIR } & \begin{array}{l}\text { Data from the Epidemiological Study on the } \\ \text { Insulin Resistance Syndrome }\end{array} \\ \text { FPG } & \text { Fasting plasma glucose } \\ \text { GWAS } & \text { Genome-wide association studies } \\ \text { HIT } & \text { Hamster insulinoma tumour } \\ \text { MAGIC } & \text { Meta-Analyses of Glucose and Insulin-related } \\ & \text { Traits Consortium } \\ \text { RSV } & \text { Rous sarcoma virus } \\ \text { SNP } & \text { Single nucleotide polymorphism } \\ \text { USF } & \text { Upstream stimulatory factor }\end{array}$

\section{Introduction}

The glucose-6-phosphatase catalytic subunit gene family comprises three members, G6PC, G6PC2 and G6PC3 [1]. All three catalyse the hydrolysis of glucose-6-phosphate to glucose and inorganic phosphate, although with markedly different kinetics [1]. The G6PC2 gene, also known as $I G R P$ [2-4], is principally expressed in the beta cells of pancreatic islets [5]. Recent genome-wide association studies (GWAS) in humans have identified genetic association signals in and near G6PC2 that are associated with variations in fasting plasma glucose (FPG) and $\mathrm{HbA}_{1 \mathrm{c}}$ levels $[6$, 7]. FPG and $\mathrm{HbA}_{1 \mathrm{c}}$ are important metabolic traits that are correlated with the risk of developing type 2 diabetes [8-10] and with cardiovascular-associated mortality [11-13]. Many other studies have confirmed that the G6PC2 locus harbours the strongest common genetic determinant of FPG levels in terms of significance and effect size, particularly with a common single nucleotide polymorphism (SNP), rs560887, that is located in the third intron of G6PC2, and that explains around $1 \%$ of the total variance in FPG [14-18]. These genetic data are consistent with the approximately $15 \%$ decrease in FPG observed following a global knockout of $G 6 p c 2$ in mice [4] and support the hypothesis that genetic variation within the G6PC2 gene, rather than the surrounding genes, directly contributes to variations in FPG in humans. These findings suggest that G6PC2 may regulate FPG by opposing the action of glucokinase, thereby modulating beta cell glycolytic flux and glucose-stimulated insulin secretion [19].

A key step in extending the results of these GWAS is establishing a functional link between specific common SNPs in the G6PC2 locus and variations in FPG. In a previous study, we demonstrated that two common G6PC2 promoter SNPs, rs13431652 and rs573225, are associated with FPG levels as part of the same association signal as rs560887 [20]. For SNP rs13431652, the A allele was associated with increased FPG and elevated promoter activity, consistent with the putative function of G6PC2 in pancreatic islets [20]. In contrast, for SNP rs573225, the A allele was associated with increased FPG but reduced promoter activity, at odds with the putative function of G6PC2 in pancreatic islets [20]. These data suggested that rs13431652 is a potentially causative SNP whereas rs573225 is a functional SNP that opposes the action of causative SNPs [20]. Because the rs573225-A allele negates the effect of the rs13431652-A allele [20], the data also suggested that the G6PC2 locus must contain additional causative SNPs. The goal of the experiments described here was to identify potentially causative SNPs using a combination of genetics and molecular studies. Specifically, we assessed the potential of the intronic SNP rs560887 to alter G6PC2 RNA splicing. In addition, we examined the contribution of an additional common G6PC2 promoter SNP, rs2232316, to the association signal as well as its effect on transcription factor binding and G6PC2 fusion gene expression.

\section{Methods}

Study participants The Data from the Epidemiological Study on the Insulin Resistance Syndrome (DESIR) cohort is a longitudinal French general population cohort and is fully described elsewhere [6]. We analysed 4,220 DESIR participants with normal FPG (defined as FPG $<6.1 \mathrm{mmol} / 1$ without hypoglycaemic treatment) who were successfully genotyped. The study protocol was approved by the ethics committee of Bicêtre Hospital (Paris, France).

We also used publicly available datasets from a meta-analysis of European GWAS for FPG levels, which was performed by the Meta-Analyses of Glucose and Insulin-related Traits Consortium (MAGIC; www.magicinvestigators.org), with a total of 46,186 non-diabetic participants [17].

A full list of DESIR and MAGIC investigators is provided in the Electronic supplementary material (ESM). 
Genotyping of SNP rs2232316 Genotyping of SNP rs2232316 was performed using a custom designed iSELECT array (Metabochip, Illumina, Santa Clara, CA, USA; ref no.: chr2:169465808 [NCBI36/hg18]), according to the manufacturer's instructions. This array contains around 200,000 SNPs and was developed to support the large-scale follow-up of putative associations for metabolic traits and fine-mapping studies of GWAS-identified loci [21]. The genotyping success rate of SNP rs2232316 was $99 \%$. No significant deviation $(p>0.05)$ from HardyWeinberg equilibrium was observed.

Genotyping of SNPs rs560887 and rs573225 has previously been reported $[6,20]$.

Minigene construction and splicing analyses Minigenes were constructed in the RHCglo minigene vector, a generous gift from T. Cooper (Baylor College of Medicine, Houston, TX, USA) [22]. This vector contains the Rous sarcoma virus (RSV) promoter, a 5' exon with a splice junction, an artificial exon surrounded by intron sequences from the human beta-globin gene and a $3^{\prime}$ exon with a splice junction
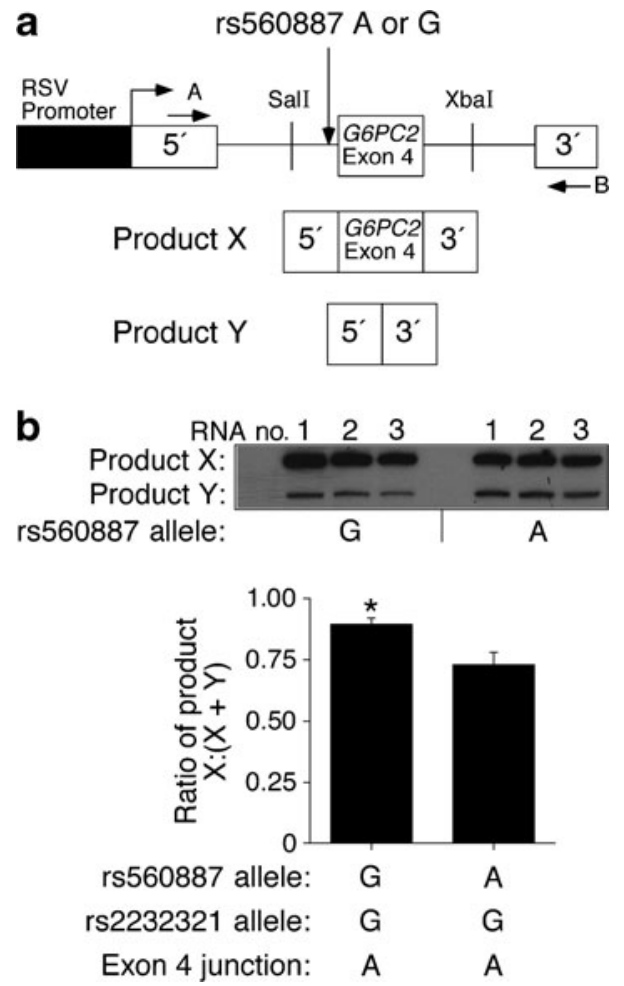

Fig. 1 rs560887 alters $G 6 P C 2$ exon 4 splicing. (a) The RHCglo minigene vector. The vector comprises the RSV promoter, a $5^{\prime}$ exon, a $3^{\prime}$ exon and Sall plus XbaI restriction enzyme sites into which G6PC2 exon (E) 4 was ligated along with surrounding $5^{\prime}$ and $3^{\prime}$ intronic sequences. RT-PCR was used to quantify the relative abundance of splice products $\mathrm{X}$ and $\mathrm{Y}$ using primers A and $\mathrm{B}$. (b) RHCglo minigene vectors containing the alternate alleles of rs560887 were transiently transfected into HeLa cells and RNA expression was quantified as described in the Methods. Results show the mean data from three experiments \pm SEM. ${ }^{*} p<0.05$ vs the rs $560887-A$ allele
(Fig. 1a) [22]. The artificial exon and surrounding human beta-globin gene intron sequences were replaced by $G 6 P C 2$ exon 4 and approximately $300 \mathrm{bp}$ of surrounding intronic sequences. Two minigenes were constructed containing the alternate alleles of rs 560887 .

These minigenes were expressed in tissue culture cells using transient transfection with RNA isolated $24 \mathrm{~h}$ post-transfection. cDNA was then generated using reverse transcriptase in conjunction with a primer complementary to the $3^{\prime}$ vector exon (Fig. 1a; primer B). Using primer A in combination with primer B (Fig. 1a), splicing between the vector $5^{\prime}$ and $3^{\prime}$ exons and the inserted $G 6 P C 2$ exon was assessed. PCR reactions were performed under conditions where amplification was in the linear range (ESM Fig. 1), and the products (X and Y; Fig. 1a) were then resolved on non-denaturing polyacrylamide gels as previously described [23]. DNA sequencing confirmed the identity of these products and the presence of the predicted splice junctions. See ESM Methods for a detailed description.

Fusion gene plasmid construction The originally isolated G6PC2 promoter contained the rs2232316-G and rs573225-A alleles [3]. The construction of a human G6PC2-luciferase fusion gene containing promoter sequence from -324 to +3 has been previously described [20]. A three-step PCR strategy [24] was used to introduce the alternate rs2232316-A and/or rs573225-G alleles into the human $G 6 P C 2$ promoter within the context of the -324 to +3 promoter fragment. Promoter fragments generated by PCR were completely sequenced to ensure the absence of polymerase errors. All plasmid constructs were purified by centrifugation through caesium chloride gradients.

Cell culture, transient transfection and luciferase assays Hamster insulinoma tumour (HIT), $\beta$ TC-3 and MIN6 cells were cultured and transfected with various $G 6 P C 2-$ luciferase fusion genes in the pGL3 MOD vector $(2 \mu \mathrm{g})$ and an expression vector encoding Renilla luciferase $(0.5 \mu \mathrm{g})$ using lipofectamine as previously described [3, 25]. Following transfection, cells were incubated for $18-20 \mathrm{~h}$ in serum-containing medium. The cells were then harvested, and firefly and Renilla luciferase activity were assayed as previously described [25].

Gel retardation assays The analysis of Foxa2 binding using gel retardation assays was performed exactly as previously described [20]. See ESM Methods for a detailed description.

Statistical analysis For genetic studies the effect of SNPs on FPG levels was assessed using linear regression models adjusted for age, sex and BMI, or for age, sex, BMI and other G6PC2 SNPs (rs573225 and/or rs560887) in conditional regression, under the additive model. All statistical analyses of genetic data were performed with IBM SPSS 
software (version 14; Armonk, NY, USA). Of note, the MAGIC consortium used linear regressions adjusted for age and sex only [17].

For functional studies the transfection data were analysed for differences from the control values, as specified in the figures. Statistical comparisons were calculated using an unpaired Student's $t$ test. The level of significance was taken to be $p<0.05$ (two-sided test).

\section{Results}

SNP rs560887 alters G6PC2 RNA splicing Previous studies have shown that multiple $G 6 P C 2$ splicing isoforms exist in human islets, with a variant that lacks exon 4 being the most prominent $[3,26]$. SNP rs560887 is located 26 nucleotides $5^{\prime}$ of G6PC2 exon 4. A minigene strategy, in which splicing of individual exons can be assessed [27, 28], was used to address the hypothesis that rs560887 affects G6PC2 RNA splicing (Fig. 1a). Figure 1b shows that the alternate alleles of rs560887 alter the relative abundance of splicing products $\mathrm{X}$ and $\mathrm{Y}$ (Fig. 1a), with the rs560887-G allele promoting improved splicing, specifically the inclusion of G6PC2 exon 4 . This is consistent with the genetic association between the rs560887-G allele, elevated FPG and the putative function of G6PC2, suggesting that rs560887 is a potentially causative SNP.

The genetic variant rs2232321 also alters G6PC2 RNA splicing Another genetic variant, rs2232321, is located immediately downstream of rs560887, and we hypothesised that this variant might also affect splicing. Using the same minigene strategy as described above, Fig. 2 shows that the

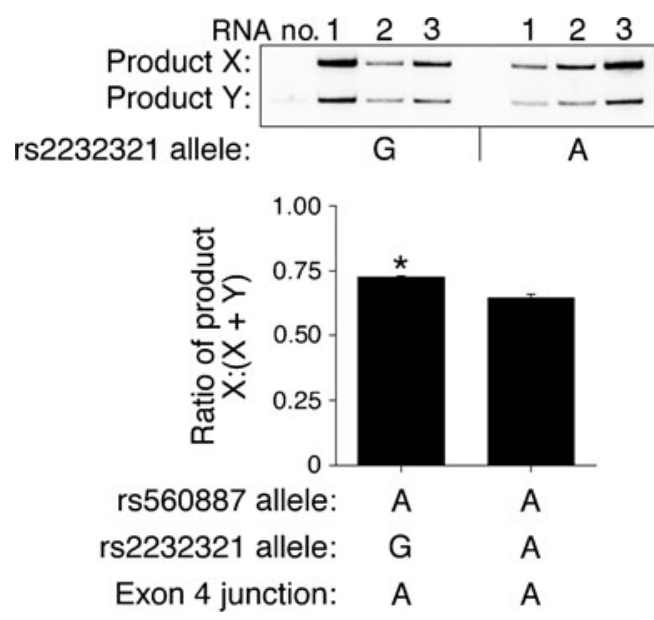

Fig. 2 rs 2232321 alters G6PC2 RNA splicing. RHCglo minigene vectors containing the alternative alleles of rs2232321 were transiently transfected into HeLa cells, and RNA expression was quantified as described in the Methods. Results show the mean data from three experiments \pm SEM. ${ }^{*} p<0.05$ vs the rs 2232321 -A allele alternate alleles of rs2232321 alter the relative abundance of products $\mathrm{X}$ and $\mathrm{Y}$, with the rs2232321-G allele promoting inclusion of G6PC2 exon 4, although the magnitude of the effect was smaller than seen for rs560887 (compare Fig. 1b and Fig. 2).

The effects of rs560887 and rs2232321 are dependent on the non-consensus exon 4 splice junction We have previously shown that, in both mice and humans, the $5^{\prime}$ exon 4 splice junction contains the non-consensus sequence tag/A [3,29] instead of cag/G [30] (the intronic sequence being in lower case letters). We hypothesised that the presence of this nonconsensus splice junction might result in splicing being more susceptible to the influence of rs560887 and rs2232321. This hypothesis was tested using the same minigene approach as discussed above. Figure 3 shows that when the sequence of the non-consensus $5^{\prime}$ exon 4 splice junction is converted to tag $/ \mathrm{G}$, the inclusion of $G 6 P C 2$ exon 4 is markedly enhanced, despite the presence of the suboptimal rs560887-A allele. This indicates that the influence of the alternate alleles of rs 560887 and rs 2232321 on G6PC2 RNA splicing is, quantitatively, strongly dependent on the presence of the non-consensus exon 4 splice junction.

SNP rs2232316 alters G6PC2 fusion gene expression in $\beta T C-3$ cells We previously demonstrated that the -306 to +3 region of the mouse $G 6 p c 2$ promoter confers high fusion gene expression in islet-derived cell lines $[24,29,31]$ and is sufficient to initiate transgene expression in mouse islets, predominantly in beta cells, at the expected time in development, around embryonic day 14 [32]. In addition, this region is highly conserved in the human $G 6 P C 2$ promoter

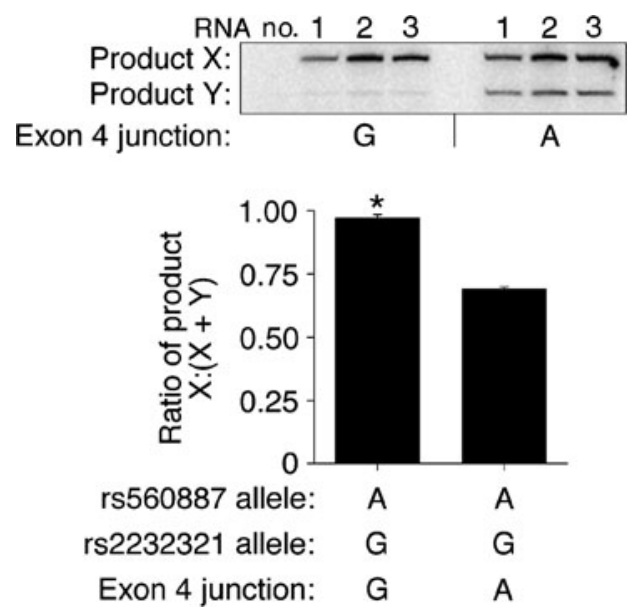

Fig. 3 The effects of rs560887 and rs2232321 are dependent on the non-consensus exon 4 splice junction. RHCglo minigene vectors containing the native (A) or consensus $(\mathrm{G})$ exon $45^{\prime}$ splice junction were transiently transfected into HeLa cells and RNA expression was quantified as described in the Methods. Results show the mean data from three experiments \pm SEM. ${ }^{*} p<0.05$ vs the exon 4 junction A allele 
[3], which likewise confers high fusion gene expression in islet-derived cell lines (ESM Fig. 2), although not in HeLa or hepatoma cells [33]. To identify potentially causative SNPs that affect $G 6 P C 2$ transcription, we therefore focused on this promoter region. A common SNP in this region, rs 2232316 , located at -238 , relative to the transcription start site [29], lies within conserved stretches of nucleotides that have the potential to represent an important cis-acting element [3]. We therefore investigated whether rs 2232316 could affect $G 6 P C 2$ fusion gene expression. Figure 4 shows the analysis, by transient transfection of islet-derived $\beta$ TC3, HIT and MIN6 cells, of fusion genes containing each of the rs2232316 alleles, generated in the context of the -324 to +3 G6PC2 promoter region. The data show that the rs2232316-A allele was associated with an approximately $50-100 \%$ increase in promoter activity in comparison to that observed with the rs2232316-G allele (Fig. 4).

SNP rs2232316 alters Foxa2 binding to the G6PC2 promoter in vitro We next investigated whether the alternate alleles of rs2232316 affected transcription factor binding. The G6PC2 promoter region that encompasses rs 2232316 was analysed using MatInspector sequence analysis software (Genomatix Software, Munich, Germany) [34] with the goal of identifying a cis-acting element whose binding of its cognate trans-acting factor was likely to be affected by the alternate rs2232316 alleles. This analysis identified a forkhead transcription factor binding motif [35].

While several members of the Fox family are expressed in the pancreas [35], members of the Foxa subgroup have been shown to play important roles in islet development and function [36]. Gel retardation assays were used to investigate whether any of the three Foxa isoforms [36] could bind to this $G 6 P C 2$ promoter region in vitro. When a labelled double-stranded oligonucleotide, designated rs2232316-A, representing the $G 6 P C 2$ promoter sequence from -250 to -226 and containing the rs2232316-A allele (Fig. 5a), was incubated with nuclear extract prepared from $\beta$ TC- 3 cells, a single protein-DNA complex was detected (Fig. 5b; lanes 1 and 4). To identify the factor present in this complex, a gel retardation assay was performed in which $\beta$ TC-3 cell nuclear extract was preincubated with antisera specific for Foxa2 (lane 2) or, as a control, upstream stimulatory factor 2 (USF-2) (lane 3). Previous studies have shown that Foxa2 is the predominant Foxa isoform in $\beta$ TC-3 cells [25]. As can be seen in Fig. 5b (arrow), the addition of antibodies recognising Foxa2 resulted in a clear supershift in the migration of the complex, whereas the addition of antibodies recognising USF-2 had no effect. This result strongly suggests that the complex represents Foxa2 binding.

Gel retardation competition experiments, in which a varying molar excess of unlabelled oligonucleotide was included with the labelled rs2232316-A oligonucleotide, were used to compare the affinity of Foxa2 binding to the rs2232316 variants of this G6PC2 Foxa2 binding site. Figure 6(a,b) shows that the rs2232316-A oligonucleotide competed effectively for the formation of the Foxa2-DNA complex, whereas the rs2232316-G oligonucleotide did not. Consistent with the competition experiment data (Fig. 6a, b), the direct analysis of Foxa2 binding to the rs2232316-A and -G oligonucleotide probes, labelled with the identical specific activity, showed a dramatic difference in Foxa2 binding affinity (Fig. 6c). Interestingly, the original consensus sequence for Foxa2 binding (Fig. 5A) [37] indicates that either an A or a $\mathrm{G}$ nucleotide at the location of the rs2232316 SNP will support Foxa2 binding, but clearly, for this particular Foxa2 binding site, this is not the case (Fig. 6c). This consensus was established using a sequential selection and amplification of binding sites protocol [37]. In contrast, an analysis of endogenous Foxa2 binding sites based on ChIP-Seq data [38] indicates that an A nucleotide is preferred at the location of the rs2232316 SNP in vivo, consistent with the binding data (Fig. 6). The preference for the A nucleotide was evident under multiple distinct assay conditions (Fig. 6c).

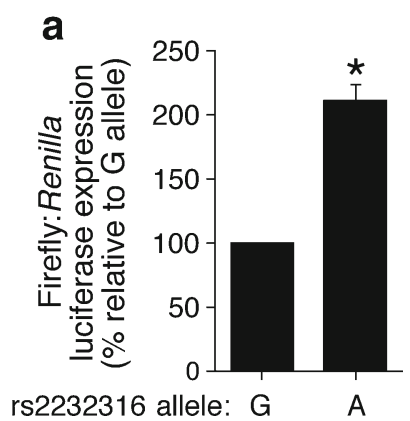

Fig. 4 The human G6PC2 rs2232316-A allele is associated with increased $G 6 P C 2$ promoter activity relative to the rs2232316-G allele. $\beta \mathrm{TC}-3$ (a), HIT (b) and MIN6 (c) cells were transiently co-transfected with various $G 6 P C 2-$ luciferase fusion genes and an expression vector encoding Renilla luciferase as described in the Methods. The G6PC2luciferase fusion genes represented the rs2232316-A or -G alleles present
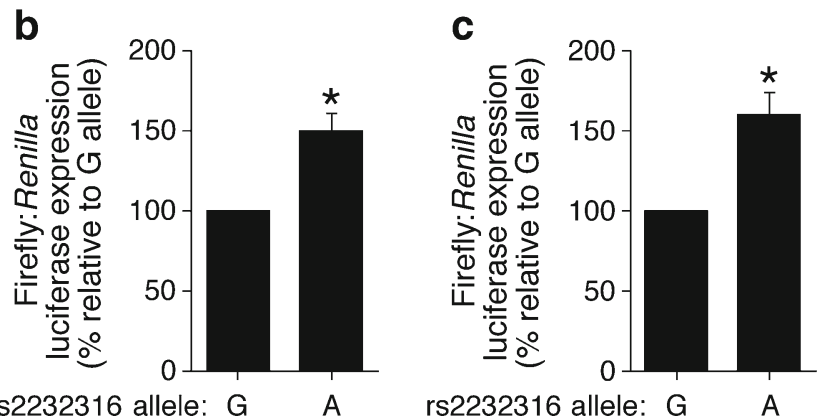

in the context of the human G6PC2 promoter sequence located between -324 and +3 . Results are presented as the ratio of firefly:Renilla luciferase activity, expressed as a percentage relative to the value obtained with the rs $2232316-\mathrm{G}$ allele, and represent the mean of three experiments \pm SEM, each using an independent preparation of each fusion gene plasmid, assayed in triplicate. ${ }^{*} p<0.05$ vs the rs $2232316-\mathrm{G}$ allele 


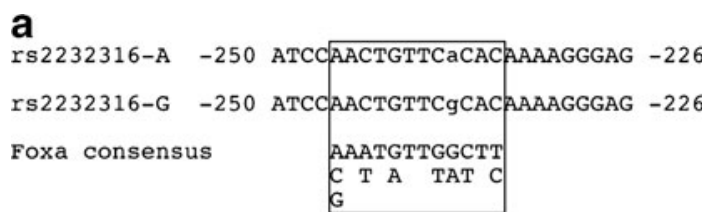

b



Fig. 5 Foxa2 binds the human $G 6 P C 2-250 /-226$ promoter region in vitro. (a) The sense strand sequences of the oligonucleotides used in gel retardation assays are shown. SNP base pairs are shown in bold lower case letters. The consensus Foxa binding motif, $(\mathrm{A} / \mathrm{G}) \mathrm{A}(\mathrm{G} / \mathrm{A})(\mathrm{C} /$ $\mathrm{T})(\mathrm{C} / \mathrm{A}) \mathrm{AA}(\mathrm{C} / \mathrm{T}) \mathrm{A}(\mathrm{T} / \mathrm{A}) \mathrm{T}(\mathrm{T} / \mathrm{G} / \mathrm{C})$, is taken from [37]. (b) $\beta \mathrm{TC}-3 \mathrm{nu}-$ clear extract was incubated in the absence (none) or presence of the indicated antiserum for $10 \mathrm{~min}$ on ice. Labelled oligonucleotides representing the $-250 /-226 G 6 P C 2$ promoter region and containing the rs2232316-A allele (rs2232316-A; a) were then added and incubation continued for $20 \mathrm{~min}$ at room temperature. Protein binding was then analysed using the gel retardation assay as described in the Methods. In the representative audioradiograph shown, only the retarded complexes are visible and not the free probe, which was present in excess. A single major complex was detected that represents Foxa2 binding. The arrow indicates a supershifted complex

SNP rs2232316 does not influence the effect of SNP rs573225 on G6PC2 fusion gene expression in $\beta T C-3$ cells We recently showed that the alternate alleles of rs573225 also alter Foxa2 binding to the G6PC2 promoter in vitro [20]. The Foxa2 binding sites that encompass rs573225 (-261/-250) and rs2232316 (-246/-235) are located three nucleotides apart in the G6PC2 promoter [3], and this region is highly conserved in the mouse G6pc2 promoter [3]. Because the Foxa2 binding sites that encompass rs573225 and rs2232316 are so close, we hypothesised that Foxa2 binding at one element might modulate the action of Foxa2 at the other. The interaction between these SNPs on G6PC2 fusion gene expression was therefore investigated (Fig. 7). The results show that the rs573225-G allele and the rs2232316-A allele have an additive and, therefore, apparently independent effect on fusion gene expression (Fig. 7).

SNP rs2232316 is associated with FPG levels but not independently from SNPs rs573225 or rs560887 We next assessed the association of SNP rs2232316 with FPG levels in 4,220 individuals with normal FPG $(<6.1 \mathrm{mmol} / \mathrm{l})$ from the French DESIR cohort. We found that the minor rs2232316-A allele associates with increased FPG levels $(\beta=0.040 \mathrm{mmol} / 1$, $\boldsymbol{p}=4.3 \times 10^{-3}$ ) (Table 1$)$. By using publicly available datasets from the MAGIC consortium, we found that the association between the rs2232316-A allele and increased FPG levels was also highly significant in 46,186 non-diabetic participants $\left(\beta=0.04 \mathrm{mmol} / \mathrm{l}, p=7.3 \times 10^{-10}\right)$ [17].

Conditioned regression model analyses in DESIR to assess the independency from SNPs rs573225 or rs560887 showed a lack of significance of rs2232316 when we took into account the effect of rs573225 or rs560887 $(p=0.29$ and $p=0.35$, respectively; Table 1), whereas the effects of SNPs rs 573225 or rs 560887 were unchanged $\left(\beta=0.076 \mathrm{mmol} / 1, p=2.7 \times 10^{-14}\right.$

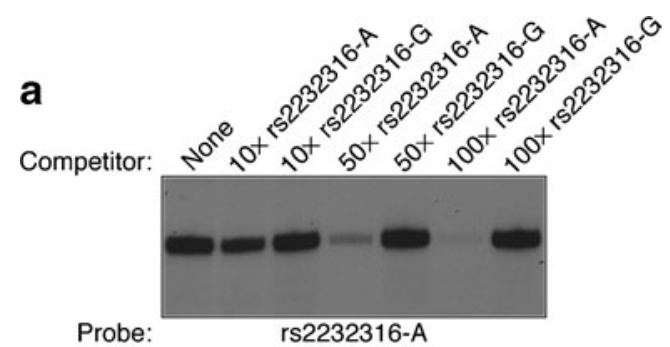

b

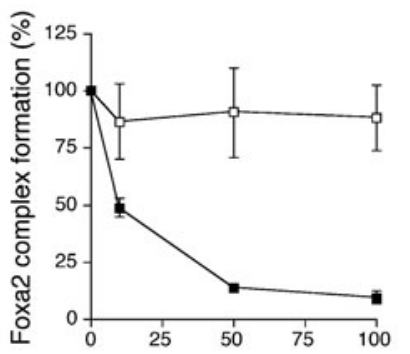

Molar excess of competitor DNA

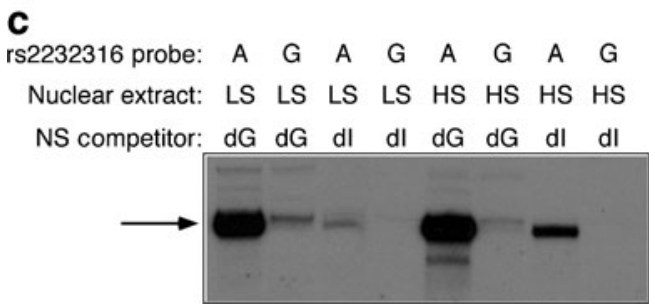

Fig. 6 Comparison of the effect of the rs2232316-A and -G alleles on Foxa2 binding affinity in vitro. (a) Labelled rs2232316-A oligonucleotides, (Fig. 5a) were incubated in the absence or presence of the indicated molar excess of the unlabelled human rs2232316-A or -G oligonucleotide competitors (Fig. 5a) prior to the addition of $\beta$ TC-3 cell nuclear extract. Protein binding was then analysed using the gel retardation assay as described in the Methods. In the representative autoradiograph shown, only the retarded complex is visible and not the free probe, which was present in excess. (b) Protein binding, specifically ${ }^{32} \mathrm{P}$ associated with the retarded complex, was quantified as described in the Methods. The data represents the mean \pm SEM of three experiments. Closed squares, rs2232316-A oligonucleotide; open squares, rs2232316-G oligonucleotide. (c) The labelled rs2232316-A and -G oligonucleotides (Fig. 5a) were incubated with either high salt (HS) or low salt (LS) $\beta$ TC-3 cell nuclear extract and in the presence of either the poly (dI-dC).poly(dI-dC) (dI) or poly(dG-dC).poly(dG-dC) (dG) non-specific competitors, which influence the strength of Foxa 2 binding [50]. Protein binding was then analysed using the gel retardation assay as described in the Methods. The arrow points to the specific Foxa2-DNA complex that preferentially forms with the rs2232316-A oligonucleotides 


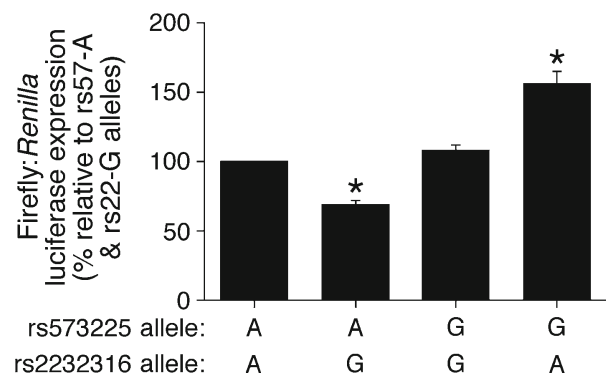

Fig. 7 The rs2232316 and rs573225 SNPs have independent effects on G6PC2 promoter activity in $\beta \mathrm{TC}-3$ cells. $\beta \mathrm{TC}-3$ cells were transiently co-transfected with various $G 6 P C 2$-luciferase fusion genes and an expression vector encoding Renilla luciferase as described in the Methods. The G6PC2-luciferase fusion genes represented the rs2232316-A or -G alleles and rs573225-A or $-\mathrm{G}$ alleles present in the context of the human G6PC2 promoter sequence located between -324 and +3 . Results are presented as the ratio of firefly:Renilla luciferase activity, expressed as a percentage relative to the value obtained with the rs2232316-A and rs573225-A alleles, and represent the mean of three experiments \pm SEM, each using an independent preparation of each fusion gene plasmid, assayed in triplicate. ${ }^{*} p<0.05$ vs (rs2232316-G and rs573225-A) and (rs2232316-A and rs573225-G) alleles

and $\beta=0.084 \mathrm{mmol} / 1, p=6.8 \times 10^{-17}$, respectively; Table 1 ). Of note, despite a low correlation between rs2232316 and rs573225 or rs560887 $\left(r^{2}=0.066\right.$ and $r^{2}=0.046$, respectively; in DESIR) due to marked differences in frequency of the effect alleles (Table 1), we observed a very high linkage disequilibrium between rs2232316 and rs573225 or rs560887 (D'>0.95), indicating that the rs2232316 association signal with FPG levels is not independent of association signals for SNPs rs573225 or rs560887. When we added SNPs rs2232316, rs573235 and rs560887 into the same linear regression model, the effects of SNPs rs2232316 and rs573235 on FPG levels were not significant ( $p=0.22$ and $p=0.95$, respectively; Table 1), whereas the effect of the rs560887-G allele on increased FPG levels was unchanged ( $\beta=0.084 \mathrm{mmol} / \mathrm{l}, p=2.0 \times 10^{-3}$; Table 1$)$. Therefore, the intronic SNP rs560887 seems to drive the association with FPG levels in the DESIR cohort. However, it will be important to repeat these genetic analyses in other populations.

\section{Discussion}

GWAS have recently provided important new insights about the genetics of common forms of type 2 diabetes and some of its related quantitative traits such as FPG and $\mathrm{HbA}_{1 \mathrm{c}}[6,7$, 14-18, 39-42]. The studies described here represent a key step in extending these discovery-phase GWAS data through

Table 1 Association between G6PC2 SNPs and FPG levels in 4,220 normoglycaemic French participants from the DESIR study

\begin{tabular}{|c|c|c|c|c|c|c|c|}
\hline SNP & Adjustments & $\begin{array}{l}\text { Frequency of the } \\
\text { allele increasing } \\
\text { FPG (Y) }(\%)\end{array}$ & $\begin{array}{l}\text { Mean FPG level } \\
\text { by genotype } \\
(\mathrm{mmol} / \mathrm{l}) \mathrm{XX}\end{array}$ & $\begin{array}{l}\text { Mean FPG level } \\
\text { by genotype } \\
(\mathrm{mmol} / \mathrm{l}) \mathrm{XY}\end{array}$ & $\begin{array}{l}\text { Mean FPG level } \\
\text { by genotype } \\
(\mathrm{mmol} / \mathrm{l}) \mathrm{YY}\end{array}$ & $\beta(\mathrm{SE})^{\mathrm{a}}$ & $p$ value \\
\hline $\begin{array}{l}\text { rs2232316 X-allele }=\mathrm{G} \\
\quad \text { Y-allele }=\mathrm{A}\end{array}$ & Age, sex, BMI & 11.4 & $5.19 \pm 0.45$ & $5.25 \pm 0.43$ & $5.22 \pm 0.46$ & $0.040(0.1)$ & $4.3 \times 10^{-3}$ \\
\hline $\begin{array}{l}\text { rs573225 X-allele }=\mathrm{G} \\
\text { Y-allele }=\mathrm{A}\end{array}$ & Age, sex, BMI & 67.0 & $5.11 \pm 0.45$ & $5.17 \pm 0.46$ & $5.25 \pm 0.44$ & $0.079(0.01)$ & $2.8 \times 10^{-16}$ \\
\hline $\begin{array}{l}\text { rs560887 X-allele }=A \\
\quad \text { Y-allele }=\mathrm{G}\end{array}$ & Age, sex, BMI & 69.5 & $5.09 \pm 0.45$ & $5.16 \pm 0.46$ & $5.25 \pm 0.44$ & $0.086(0.01)$ & $1.8 \times 10^{-18}$ \\
\hline $\begin{array}{l}\text { rs2232316 X-allele }=G \\
\text { Y-allele }=\mathrm{A}\end{array}$ & $\begin{array}{l}\text { Age, sex, BMI, } \\
\text { rs } 573225\end{array}$ & 11.4 & NA & NA & NA & $0.016(0.01)$ & 0.29 \\
\hline $\begin{array}{l}\text { rs573225 X-allele }=\mathrm{G} \\
\quad \text { Y-allele }=\mathrm{A}\end{array}$ & $\begin{array}{l}\text { Age, sex, BMI, } \\
\text { rs } 2232316\end{array}$ & 67.0 & NA & NA & NA & $0.076(0.01)$ & $4.0 \times 10^{-14}$ \\
\hline $\begin{array}{l}\text { rs2232316 X-allele }=G \\
\text { Y-allele }=\mathrm{A}\end{array}$ & $\begin{array}{l}\text { Age, sex, BMI, } \\
\text { rs } 560887\end{array}$ & 11.4 & NA & NA & NA & $0.014(0.01)$ & 0.35 \\
\hline $\begin{array}{l}\text { rs560887 X-allele }=A \\
\quad \text { Y-allele }=\mathrm{G}\end{array}$ & $\begin{array}{l}\text { Age, sex, BMI, } \\
\text { rs } 2232316\end{array}$ & 69.5 & NA & NA & NA & $0.083(0.01)$ & $2.9 \times 10^{-16}$ \\
\hline $\begin{array}{l}\text { rs2232316 X-allele }=\mathrm{G} \\
\quad \text { Y-allele }=\mathrm{A}\end{array}$ & $\begin{array}{l}\text { Age, sex, BMI, } \\
\text { rs573225, } \\
\text { rs560887 }\end{array}$ & 11.4 & NA & NA & NA & $0.018(0.01)$ & 0.22 \\
\hline $\begin{array}{l}\text { rs573225 X-allele }=\mathrm{G} \\
\text { Y-allele }=\mathrm{A}\end{array}$ & $\begin{array}{l}\text { Age, sex, BMI, } \\
\text { rs2232316, } \\
\text { rs560887 }\end{array}$ & 67.0 & NA & NA & NA & $0.000(0.03)$ & 0.99 \\
\hline $\begin{array}{l}\text { rs560887 X-allele }=A \\
\quad \text { Y-allele }=G\end{array}$ & $\begin{array}{l}\text { Age, sex, BMI, } \\
\text { rs573225, } \\
\text { rs2232316 }\end{array}$ & 69.5 & NA & NA & NA & $0.082(0.03)$ & $2.5 \times 10^{-3}$ \\
\hline
\end{tabular}

Data are presented as mean $\pm \mathrm{SD}$

${ }^{a}$ Per Y-allele effect size: coefficient $\beta$ from additive linear regression models adjusted for age, sex and BMI, or for age, sex, BMI and other G6PC2 SNPs in conditional regressions

NA, not applicable 
the analysis of the functional properties of variants that span the G6PC2 locus (ESM Fig. 3). Using minigenes, in which splicing of individual exons can be assessed [27, 28], we show that a common variant, rs560887, located in the third intron of $G 6 P C 2$, modulates $G 6 P C 2$ pre-RNA splicing (Fig. 1), as does a rare variant, rs2232321, located immediately $3^{\prime}$ of rs560887 (Fig. 2). Interestingly, the functional effect of these variants is absolutely contingent on the presence of a non-consensus splice junction (Fig. 3). In addition, using fusion genes, we show that another common variant, rs2232316, affects $G 6 P C 2$ promoter activity (Fig. 4) by modulating Foxa2 binding (Figs 5 and 6). For both common SNPs, rs560887 and rs2232316, the in situ functional data are in accord with the putative function of G6PC2 in pancreatic islets in that the allele associated with elevated G6PC2 expression is genetically associated with elevated FPG (Table 1) [6], indicating that both variants are potentially causative. Thus, based on the analysis of G6pc2 knockout mice [4] and the resulting hypothesis that G6PC2 opposes the action of glucokinase, elevated $G 6 P C 2$ expression is predicted to lead to elevated FPG.

This current study, combined with our previous analysis of the rs573225 and rs13431652 promoter SNPs [20], highlights several limitations in identifying causative SNPs. First, because rs560887, rs2232316 and rs13431652 are all part of the same association signal and because of differences between populations [20], we cannot definitively determine whether one or all of these SNPs are truly causative. Second, we would ideally like to obtain additional support for our conclusions through an analysis of endogenous $G 6 P C 2$ expression in pancreatic islets; however, previous experience has shown that the limited sample sizes available from human cadavers are insufficient to detect significant correlations [20]. This appears to be because SNPs in the G6PC2 gene have relatively subtle effects on G6PC2 expression (Figs $1 \mathrm{~b}$ and 4) [20]. This contrasts with similar studies examining SNPs in the TCF7L2 gene, in which significant correlations between genotype and islet expression have been detected because these SNPs markedly alter TCF7L2 gene expression [43, 44]. Finally, as previously noted [20], confidence in the results of promoter SNP analyses is always tempered by the multiple caveats associated with analysing $G 6 P C 2$ fusion gene transcription in islet-derived cell lines. We attempted to analyse the effect of these SNPs on G6PC2 fusion gene transcription in primary islet cells, but expression was below the limit of detection (data not shown). Similarly, we attempted to repeat the splicing analyses in the islet beta cell-derived $\beta \mathrm{TC}-3$ and HIT cell lines, but minigene expression was below the limit of detection (data not shown). Instead, HeLa cells were used for the splicing analyses due to their high transfection efficiency. As such, further studies will be required to demonstrate that the results obtained when analysing promoter and intronic SNPs using cell lines are relevant to their true effects on endogenous gene expression in vivo. We can be more confident that rs560887 is a potentially causative SNP, in comparison with the promoter SNPs, because splicing is largely mediated by ubiquitous factors $[45,46]$ in contrast to G6PC2 gene transcription, a process mediated by beta cell-enriched transcription factors whose expression may vary between cell lines and islets [1].

The observation that the rs573225-A allele negates the effect of the rs2232316-A (Fig. 7), just as it negates the effect of the rs13431652-A allele [20], supports the concept that rs573225 is a functional SNP that opposes the action of causative SNPs on G6PC2 expression [20]. This conclusion contrasts with a previous study by Dos Santos et al [47], who reported that rs573225 was an epiSNP that was likely to explain the association signal between FPG and G6PC2. However, in reaching this conclusion, the authors apparently overlooked the fact that their in situ functional data with rs573225 [47], like ours, did not correlate with the putative function of $G 6 P C 2$ in pancreatic islets. Furthermore, as noted above, a clear limitation of our studies, and theirs [47], remains the high linkage disequilibrium between all four SNPs, which makes it difficult to conclusively establish a causative link between any or all of these variants in the $G 6 P C 2$ locus and variations in FPG.

SNP rs573225 has a paradoxical effect on G6PC2 fusion gene expression. A block mutation in the G6PC2 Foxa2 binding site that encompasses rs573225 abolishes Foxa2 binding and also markedly reduces fusion gene expression, indicating that Foxa2 acts an activator when bound to this site in the human G6PC2 promoter [20], as it does when bound to the equivalent site in the mouse $G 6 p c 2$ promoter [25]. Since the loss of Foxa2 binding to this site reduces $G 6 P C 2$ promoter activity and because the rs573225-G allele decreases Foxa2 binding [20], we anticipated that the rs573225$\mathrm{G}$ allele would also be associated with reduced $G 6 P C 2$ promoter activity. Surprisingly, the rs573225-G allele is associated with increased promoter activity (Fig. 7) [20]. The reason for this unexpected result is unknown, but Gao et al [48] have recently confirmed that Foxa factors also act as activators of endogenous G6pc2 expression.

In higher eukaryotes, pre-mRNA splicing is mediated by multiple cis elements in the pre-mRNA molecule. These elements comprise the branch point sequence, the polypyrimidine tract, the $5^{\prime}$ and $3^{\prime}$ splice sites and exonic/intronic splicing enhancers/silencers [45, 46]. Visual sequence analyses suggest that SNP rs560887 lies in a branch point sequence, defined as the position where intron lariats form $[45,46]$. Importantly, our hypothesis that SNP 
rs560887 affects FPG by acting in cis to directly modulate G6PC2 expression is at odds with the conclusion of Taneera et al [49] that SNP rs560887 acts in trans to modulate the expression of multiple other genes. Further studies will be required to resolve this discrepancy.

The relationship between G6PC2 and type 2 diabetes is unclear. In European populations, GWAS have linked G6PC2 to variations in FPG but not risk of the development of type 2 diabetes [17]. This is paradoxical as elevated FPG is considered to be a risk factor for type 2 diabetes [8-10]. Indeed, variations in the $G C K$ gene are linked to both FPG and risk of type 2 diabetes [17]. More recently, Taneera et al [49] reported that $G 6 P C 2$ expression was decreased in islets from donors with type 2 diabetes. Based on the GWAS data noted above [17], we would interpret the decrease in G6PC2 expression reported by Taneera et al [49] to be a secondary event, specifically a response to the diabetic environment, rather than a causative event that contributes to the development of type 2 diabetes. Thus, based on G6pc2 knockout mouse data [4], the decrease in G6PC2 expression reported by Taneera et al [49] would lead to enhanced insulin secretion, which would make sense in terms of a compensatory attempt by unhealthy islets to maintain insulin secretion.

In summary, our study provides genetic and functional evidence supporting an important role for the intronic variant rs560887 and the promoter variant rs2232316 in addition to the promoter variant rs13431652 [20], as potentially causative SNPs that contribute to the association signal between $G 6 P C 2$ and FPG. Future genetic analyses will be required to establish the impact of rs2232321 (Fig. 2) on the variation in FPG levels. However, this variant is very rare: in 4,300 EuropeanAmerican participants, the National Heart, Lung, and Blood Institute Exome Sequencing Project database did not show any carrier of the variant, and the minor allele frequency was about $0.8 \%$ in African-Americans according to the same database. Therefore, only very large sample collections of individuals of African origin would be sufficiently powered to detect an association with FPG levels. Future functional studies will focus on the analysis of rare G6PC2 variants that have the potential to markedly affect enzyme activity.

Acknowledgements We thank T. Cooper (Baylor College of Medicine, USA) for providing RHCglo minigene vector. We thank R. Stein (Vanderbilt University, USA), S. Efrat (Tel Aviv University, Israel) and J-i Miyazaki (Osaka University, Japan) for providing the HIT, $\beta$ TC-3 and MIN6 cell lines, respectively. We also thank M. Brissova and A. Coldren (Vanderbilt University, USA) for performing islet isolations and J. Haines (Vanderbilt University, USA) for helpful discussions on genetics. We thank all the participants of the DESIR study, Y. Labrune (CNRSUMR-8199, France) for statistical analyses support, C. Dina (CNRSUMR-8090, France) and M. Marre (CHU D'Angers, France) from the DESIR study group for advice on genetic data.
Funding The Vanderbilt Islet Procurement and Analysis Core is supported by NIH grant P60 DK20593, to the Vanderbilt Diabetes Research Training Center (VDRTC), and NIH grant DK59637, to the Vanderbilt Mouse Metabolic Phenotyping Center. Research in R. M. O'Brien's laboratory was supported by NIH grants DA027002 and DK92589. D. A. Baerenwald and L. D. Pound were supported by the Vanderbilt Molecular Endocrinology Training Program grant 5T32 DK07563. Research in D. A. Jacobson's laboratory was supported by NIH grants DK081666 and DK20593. The DESIR study was supported by Inserm contracts with CNAMTS, Lilly, Novartis Pharma and SanofiAventis; by Inserm (Réseaux en Santé Publique, Interactions entre les déterminants de la santé), Cohortes Santé TGIR, the Association Diabète Risque Vasculaire, the Fédération Française de Cardiologie, La Fondation de France, ALFEDIAM, ONIVINS, Ardix Medical, Bayer Diagnostics, Becton Dickinson, Cardionics, Merck Santé, Novo Nordisk, Pierre Fabre, Roche, Topcon. The genotyping was supported by the Conseil Régional Nord-Pas-de-Calais/Fonds Européen de Développement Economique et Régional: CPER axe Cartdiodiabète 2010-2011 grant to N. Bouatia-Naji The authors have no financial interests that would result in a conflict of interest with respect to this work.

Duality of interest The authors declare that there is no duality of interest associated with this manuscript.

Contribution statement DAB performed gel retardation, fusion gene and minigene analyses and drafted parts of the manuscript. BPF, OCU, JKO, LDP and NLC performed gel retardation and fusion gene and minigene analyses and revised the manuscript. $\mathrm{AB}$ and NB-N designed and performed genetic analyses and drafted parts of the manuscript. SC, $\mathrm{SL}$ and EE contributed to the genetic analyses and revised the manuscript. $\mathrm{BB}$ and OL managed the DESIR study, designed and performed genetic analyses, and revised the manuscript. PKD and DAJ designed and performed isolated islet studies and revised the manuscript. PF was responsible for the conception of the genetic analyses described in this manuscript and revised the manuscript. RMO'B was responsible for the conception of the molecular studies and drafted parts of the manuscript. All authors approved the final version.

\section{References}

1. Hutton JC, O'Brien RM (2009) The glucose-6-phosphatase catalytic subunit gene family. J Biol Chem 284:29241-29245

2. Arden SD, Zahn T, Steegers S et al (1999) Molecular cloning of a pancreatic islet-specific glucose-6-phosphatase catalytic subunitrelated protein. Diabetes 48:531-542

3. Martin CC, Bischof LJ, Bergman B et al (2001) Cloning and characterization of the human and rat islet-specific glucose-6phosphatase catalytic subunit-related protein (IGRP) genes. J Biol Chem 276:25197-25207

4. Wang Y, Martin CC, Oeser JK et al (2007) Deletion of the gene encoding the islet-specific glucose-6-phosphatase catalytic subunit-related protein autoantigen results in a mild metabolic phenotype. Diabetologia 50:774-778

5. Hutton JC, Eisenbarth GS (2003) A pancreatic beta-cell-specific homolog of glucose-6-phosphatase emerges as a major target of cell-mediated autoimmunity in diabetes. Proc Natl Acad Sci U S A 100:8626-8628

6. Bouatia-Naji N, Rocheleau G, van Lommel L et al (2008) A polymorphism within the G6PC2 gene is associated with fasting plasma glucose levels. Science 320:1085-1088

7. Chen WM, Erdos MR, Jackson AU et al (2008) Variations in the $\mathrm{G} 6 \mathrm{PC} 2 / \mathrm{ABCB} 11$ genomic region are associated with fasting glucose levels. J Clin Invest 118:2620-2628 
8. Droumaguet C, Balkau B, Simon D et al (2006) Use of HbA1c in predicting progression to diabetes in French men and women: data from an Epidemiological Study on the Insulin Resistance Syndrome (DESIR). Diabetes Care 29:1619-1625

9. Abdul-Ghani MA, DeFronzo RA (2009) Plasma glucose concentration and prediction of future risk of type 2 diabetes. Diabetes Care 32(Suppl 2):S194-S198

10. Edelman D, Olsen MK, Dudley TK, Harris AC, Oddone EZ (2004) Utility of hemoglobin A1c in predicting diabetes risk. J Gen Intern Med 19:1175-1180

11. Coutinho M, Gerstein HC, Wang Y, Yusuf S (1999) The relationship between glucose and incident cardiovascular events. A metaregression analysis of published data from 20 studies of 95,783 individuals followed for 12.4 years. Diabetes Care 22:233-240

12. Khaw KT, Wareham N, Luben R et al (2001) Glycated haemoglobin, diabetes, and mortality in men in Norfolk cohort of European prospective investigation of cancer and nutrition (EPIC-Norfolk). BMJ 322:15-18

13. Sarwar N, Gao P, Seshasai SR et al (2010) Diabetes mellitus, fasting blood glucose concentration, and risk of vascular disease: a collaborative meta-analysis of 102 prospective studies. Lancet 375:2215-2222

14. Prokopenko I, Langenberg C, Florez JC et al (2008) Variants in MTNR1B influence fasting glucose levels. Nat Genet 41:77-81

15. Bouatia-Naji N, Bonnefond A, Cavalcanti-Proenca C et al (2009) A variant near MTNR1B is associated with increased fasting plasma glucose levels and type 2 diabetes risk. Nat Genet 41:89-94

16. Reiling E, van 't Riet E, Groenewoud MJ et al (2009) Combined effects of single-nucleotide polymorphisms in GCK, GCKR, G6PC2 and MTNR1B on fasting plasma glucose and type 2 diabetes risk. Diabetologia 52:1866-1870

17. Dupuis J, Langenberg C, Prokopenko I et al (2010) New genetic loci implicated in fasting glucose homeostasis and their impact on type 2 diabetes risk. Nat Genet 42:105-116

18. Hu C, Zhang R, Wang C et al (2009) A genetic variant of G6PC2 is associated with type 2 diabetes and fasting plasma glucose level in the Chinese population. Diabetologia 52:451-456

19. Newgard CB, Lu D, Jensen MV et al (2002) Stimulus/secretion coupling factors in glucose-stimulated insulin secretion: insights gained from a multidisciplinary approach. Diabetes 51(Suppl 3): S389-S393

20. Bouatia-Naji N, Bonnefond A, Baerenwald DA et al (2010) Genetic and functional assessment of the role of the rs13431652-A and rs573225-A alleles in the G6PC2 promoter that strongly associate with elevated fasting glucose levels. Diabetes 59:2662-2671

21. Voight BF, Kang HM, Ding J et al (2012) The Metabochip, a custom genotyping array for genetic studies of metabolic, cardiovascular, and anthropometric traits. PLoS Genetics 8:e1002793

22. Singh G, Cooper TA (2006) Minigene reporter for identification and analysis of cis elements and trans factors affecting pre-mRNA splicing. Biotechniques 41:177-181

23. Cooper TA (1998) Muscle-specific splicing of a heterologous exon mediated by a single muscle-specific splicing enhancer from the cardiac troponin T gene. Mol Cell Biol 18:4519-4525

24. Martin CC, Oeser JK, O'Brien RM (2004) Differential regulation of islet-specific glucose-6-phosphatase catalytic subunit-related protein gene transcription by Pax-6 and Pdx-1. J Biol Chem 279:34277-34289

25. Martin C, Flemming B, Wang Y, Oeser J, O'Brien R (2008) Foxa2 and MafA regulate islet-specific glucose-6-phosphatase catalytic subunit-related protein (IGRP/G6PC2) gene expression. J Mol Endocrinol 41:315-328

26. Dogra RS, Vaidyanathan P, Prabakar KR, Marshall KE, Hutton JC, Pugliese A (2006) Alternative splicing of G6PC2, the gene coding for the islet-specific glucose-6-phosphatase catalytic subunit-related protein (IGRP), results in differential expression in human thymus and spleen compared with pancreas. Diabetologia 49:953-957

27. Stoss O, Stoilov P, Hartmann AM, Nayler O, Stamm S (1999) The in vivo minigene approach to analyze tissue-specific splicing. Brain Res Brain Res Protoc 4:383-394

28. Cooper TA (2005) Use of minigene systems to dissect alternative splicing elements. Methods 37:331-340

29. Ebert DH, Bischof LJ, Streeper RS et al (1999) Structure and promoter activity of an islet-specific glucose-6-phosphatase catalytic subunit-related gene. Diabetes 48:543-551

30. Jackson IJ (1991) A reappraisal of non-consensus mRNA splice sites. Nucleic Acids Res 19:3795-3798

31. Bischof LJ, Martin CC, Svitek CA et al (2001) Characterization of the mouse islet-specific glucose-6-phosphatase catalytic subunitrelated protein gene promoter by in situ footprinting. Correlation with fusion gene expression in the islet derived $\beta \mathrm{TC}-3$ and hamster insulinoma tumor cell lines. Diabetes 50:502-514

32. Frigeri C, Martin CC, Svitek CA et al (2004) The proximal isletspecific glucose-6-phosphatase catalytic subunit related protein (IGRP) autoantigen promoter is sufficient to initiate but not maintain transgene expression in mouse islets in vivo. Diabetes 53:1754-1764

33. Martin CC, Oeser JK, Svitek CA, Hunter SI, Hutton JC, O'Brien RM (2002) Identification and characterization of a human cDNA and gene encoding a ubiquitously expressed glucose-6phosphatase catalytic subunit-related protein. J Mol Endocrinol 29:205-222

34. Quandt K, Frech K, Karas H, Wingender E, Werner T (1995) MatInd and MatInspector: new fast and versatile tools for detection of consensus matches in nucleotide sequence data. Nucleic Acids Res 23:4878-4884

35. Hannenhalli S, Kaestner KH (2009) The evolution of Fox genes and their role in development and disease. Nat Rev Genet 10:233-240

36. Friedman JR, Kaestner KH (2006) The Foxa family of transcription factors in development and metabolism. Cell Mol Life Sci 63:2317-2328

37. Overdier DG, Porcella A, Costa RH (1994) The DNA-binding specificity of the hepatocyte nuclear factor 3 /forkhead domain is influenced by amino-acid residues adjacent to the recognition helix. Mol Cell Biol 14:2755-2766

38. Tuteja G, White P, Schug J, Kaestner KH (2009) Extracting transcription factor targets from ChIP-Seq data. Nucleic Acids Res 37: e113

39. Sparso T, Andersen G, Nielsen T et al (2008) The GCKR rs780094 polymorphism is associated with elevated fasting serum triacylglycerol, reduced fasting and OGTT-related insulinaemia, and reduced risk of type 2 diabetes. Diabetologia 51:70-75

40. Orho-Melander M, Melander O, Guiducci C et al (2008) Common missense variant in the glucokinase regulatory protein gene is associated with increased plasma triglyceride and C-reactive protein but lower fasting glucose concentrations. Diabetes 57:31123121

41. Pare G, Chasman DI, Parker AN et al (2008) Novel association of HK1 with glycated hemoglobin in a non-diabetic population: a genome-wide evaluation of 14,618 participants in the Women's Genome Health Study. PLoS Genetics 4:e1000312

42. Soranzo N, Sanna S, Wheeler E et al (2010) Common variants at ten genomic loci influence hemoglobin $\mathrm{A} 1 \mathrm{C}$ levels via glycemic and non-glycemic pathways. Diabetes 59:3229-3239

43. Lyssenko V, Lupi R, Marchetti P et al (2007) Mechanisms by which common variants in the TCF7L2 gene increase risk of type 2 diabetes. J Clin Invest 117:2155-2163

44. Gaulton KJ, Nammo T, Pasquali L et al (2010) A map of open chromatin in human pancreatic islets. Nat Genet 42:255-259

45. Sharp PA (1994) Split genes and RNA splicing. Cell 77:805-815

46. Solis AS, Shariat N, Patton JG (2008) Splicing fidelity, enhancers, and disease. Front Biosci 13:1926-1942 
47. Dos Santos C, Bougneres P, Fradin D (2009) An SNP in a methylatable Foxa2 binding site of the G6PC2 promoter is associated with insulin secretion in vivo and increased promoter activity in vitro. Diabetes 58:489-492

48. Gao N, Le Lay J, Qin W et al (2010) Foxa1 and Foxa2 maintain the metabolic and secretory features of the mature beta-cell. Mol Endocrinol 24:1594-1604
49. Taneera J, Lang S, Sharma A et al (2012) A systems genetics approach identifies genes and pathways for type 2 diabetes in human islets. Cell Metab 16:122-134

50. O’Brien RM, Noisin EL, Suwanichkul A et al (1995) Hepatic nuclear factor 3- and hormone-regulated expression of the phosphoenolpyruvate carboxykinase and insulin-like growth factorbinding protein 1 genes. Mol Cell Biol 15:1747-1758 